\title{
Técnica de sutura ílio-femoral com fio de polidioxanona para estabilização da articulação coxofemoral em cão com luxação coxofemoral traumática
}

\author{
Ilium-Femoral Suture Technique Using Wire of Polydioxanone \\ for Hip Joint Stabilization in a Dog with Traumatic Hip Dislocation
}

Leandro Haczkiewicz Gaiga', João Antonio Tadeu Pigatto', Carla Lempk Constantin' \& Talita Susinn²

\begin{abstract}
Background: The hip dislocation is the most common traumatic dislocation in dogs. Surgical treatment has better results, but there is still no consensus on the best technique. This study aims to report a case of traumatic hip dislocation in a dog, in which case it was decided by extra-articular stabilization, using absorbable monofilament wire of Polydioxanone, through implementing the Ilium-Femoral Suture Technique. This surgical thread is composed of a poly dioxanone polymer and differs from polyglycolic acid and polyglactin 910 by being a monofilament. The surgical technique consists of making a tunnel in the ileum body, in dorsolateral-ventromedial direction and another in the greater trochanter, in the caudally skull. The stabilization is achieved using one or more sutures standard " 8 ", passing through the two tunnels. The vast majority of published works on the subject, reports the use of non-absorbable wires, such as surgical nylon or polyamide in the making of this type of suture, justifying the relevance of this report.

Case: A Belgian Malinois breed dog of 2.5-year-old, female, was treated at a private clinic in the city of Porto Alegre, RS, Brazil, with trauma history by trampling. The patient had lack of member support and maintenance of the same in external rotation. General anesthesia of the patient, with $5 \mathrm{mg} \mathrm{kg}^{-1}$ of Propofol (Propovan ${ }^{\circledR}$ ), preceded by the application of preanesthetic medication with $0.05 \mathrm{mg} \cdot \mathrm{kg}^{-1}$ of acepromazine $0.2 \%$ (Acepran $\left.{ }^{\circledR}\right), 0.2 \mathrm{mg} \cdot \mathrm{kg}^{-1}$ of Methadone Hydrochloride $5 \%\left(\right.$ Mytedom $^{\circledR}$ ) and $3 \mathrm{mg} \cdot \mathrm{kg}^{-1}$ of Ketamine hydrochloride $10 \%$ (Quetamina ${ }^{\circledR}$ ), allowed the detailed clinical examination and proper positioning of the patient to perform the radiographic examination. The same show total loss of contact between the articular surface of the acetabulum and the femoral head rights, this being displaced skull-dorsally in relation to the acetabulum. Radiographs were compatible with dislocated hip-skull right dorsal. As an alternative to immediate treatment, we opted for the closed reduction of dislocation and making a Ehmer sling for emergency stabilization and, about $24 \mathrm{~h}$ after, extra-articular surgical stabilization by implementing the Ilium-Femoral suture technique. According to the patient's author reports, the support of the member took place on the 3rd day after surgery. An X-ray control, performed 12 weeks after the procedure, showed moderate atrophy of the muscles of the right hind limb and mild periosteal reaction in the ipsilateral ileum body region.

Discussion: The diagnosis of hip traumatic cranio-dorsal dislocation is based on patient history, clinical signs, full orthopedic examination and radiographic findings. The present patient case was an automobile trampling victim, however, the clinical signs presented as lack of support from the member and lack of external rotation may be associated with other disorders or diseases affecting the musculoskeletal system. The ease of palpation and the elevation of the greater trochanter, in relation to the ischial tuberosity and coxal and radiographs in the medial-lateral and ventral-dorsal projections made it possible to confirm the diagnosis. To prevent further tissue damage, it was held closed reduction with the patient under general anesthesia and using an Ehmer sling. Due to the size and the dog's degree of activity, we opted for the surgical stabilization. Conventional cranio-lateral approach was sufficient to expose the structures without the need for magnification to display the ileum body. The surgical technique was simple and easy to perform and the patient remained without a sling or other type of bandage for immobilization in the postoperative, an advantage of using this technique.
\end{abstract}

Keywords: dog, extra-articular absorbable suture, traumatic hip luxation.

Descritores: cão, sutura absorvível extra-articular, luxação coxofemoral traumática. 


\section{INTRODUÇÃO}

A luxação coxofemoral é a luxação traumática mais comum em pequenos animais [12]. Os acidentes automobilísticos são a causa mais comum, responsáveis por 50 a $80 \%$ dos atendimentos, sendo as quedas, brigas e incidentes desconhecidos outras causas de menor relevância [11]. Quanto ao tipo de luxação, considerando a posição da cabeça femoral em relação ao acetábulo, as mais frequentes são as crânio-dorsais, somando $90 \%$ dos casos, devido, principalmente, ao tipo de trauma e à contração dos músculos glúteos $[5,8]$. As luxações caudodorsais e ventrais ocorrem mais raramente $[1,13]$. A sutura ílio-femoral é uma técnica cirúrgica extra-articular simples, rápida, de baixo custo e que preserva as estruturas articulares, permitindo o retorno precoce da função do membro [10]. A Polidioxanona é um fio absorvível monofilamentar que mantém força tênsil por tempo prolongado e é pouco reativo aos tecidos, evitando assim, reações de corpo estranho crônicas e necessidade de nova intervenção para remoção do implante [7]. O presente relato tem por objetivo descrever a correção cirúrgica de um caso de luxação coxofemoral craniodorsal traumática em um cão (Canis familiaris), utilizando a técnica de sutura ílio-femoral com fio de Polidioxanona.

\section{CASO}

Um cão da raça Pastor Belga Malinois, com 27 $\mathrm{kg}$ de massa corporal, fêmea, de dois anos e meio de idade, foi levado ao Hospital Veterinário com histórico de trauma por atropelamento há cerca de dois dias. $\mathrm{O}$ paciente apresentava falta de apoio do membro e manutenção do mesmo em rotação externa. Estava alerta e exibia comportamento agressivo à aproximação.

Para realização do exame clínico, foi realizada contenção química com Cloridrato de Acepromazina $0,2 \%$, na dose de $0,05 \mathrm{mg} / \mathrm{kg} / \mathrm{IM}\left(\text { Acepran }^{\circledR}\right)^{1}$, associada a Cloridrato de Metadona 5\%, na dose de 0,2 mg/ $\mathrm{kg} / \mathrm{IM}\left(\text { Mytedom }^{\circledR}\right)^{2}$ e Cloridrato de Cetamina $10 \%$, na dose de $3 \mathrm{mg} / \mathrm{kg} / \mathrm{IM}\left(\text { Quetamina }^{\circledR}\right)^{1}$. Após, foi administrada Solução de Ringer com Lactato de Sódio 10 $\mathrm{mL} / \mathrm{kg} / \mathrm{h} / \mathrm{IV}\left(\text { Ringer Lactato }{ }^{\circledR}\right)^{3}$ e Propofol 1\%, na dose de $5 \mathrm{mg} / \mathrm{kg} / \mathrm{IV}\left(\operatorname{Propovan}^{\circledR}\right)^{2}$ para indução de anestesia geral. Ao exame clínico, pôde-se observar crepitação à palpação da articulação coxofemoral direita e o trocânter maior deslocado dorsalmente e mais afastado da tuberosidade isquiática em relação ao membro contralateral. Foi realizado exame radiográfico nas projeções Latero-Lateral (L-L) e Ventro-Dorsal (V-D), confirmando o diagnóstico de luxação coxofemoral craniodorsal (Figura 1a).

Prosseguiu-se com a redução fechada da luxação, obtida mediante tração e adução do membro pélvico direito, conforme descrito na literatura [13] e imobilização externa temporária com uma tipoia de Ehmer. Optou-se pela estabilização cirúrgica no dia seguinte. Foram realizados exames pré-operatórios de rotina, hemograma completo, perfil bioquímico e radiografias de tórax, para avaliação do estado geral do paciente, os quais não revelaram alterações. Foi instituído jejum pré-operatório alimentar de oito horas e hídrico de duas horas. O protocolo pré-anestésico escolhido foi Cloridrato de Acepromazina $0,05 \mathrm{mg} /$ $\mathrm{kg} / \mathrm{IM}\left(\text { Acepran }^{\circledR}\right)^{1}$ e Cloridrato de Metadona 0,2 mg/ $\mathrm{kg} / \mathrm{IM}\left(\text { Mytedom }^{\circledR}\right)^{2}$. A indução anestésica foi obtida com a administração de Propofol $5 \mathrm{mg} / \mathrm{kg} / \mathrm{IV}$ (Propo$\left.\operatorname{van}^{\circledR}\right)^{2}$ e a manutenção com Isofluorano (Isoflurano $\left.{ }^{\circledR}\right)^{4}$ ao efeito e oxigênio a $100 \%$. Como protocolo de analgesia, foram associados Cloridrato de Lidocaína $5 \mathrm{mg} / \mathrm{kg}$ (Lidovet $\left.^{\circledR}\right)^{5}$ e Sulfato de Morfina $0,1 \mathrm{mg} / \mathrm{kg}$ $\left(\text { Dolo Moff }{ }^{\circledR}\right)^{6}$, ambos administrados por via epidural. Foi utilizada Cefalotina Sódica $20 \mathrm{mg} / \mathrm{kg} / \mathrm{IV}$ (Cefariston $\left.^{\circledR}\right)^{7}$, para profilaxia antimicrobiana $30 \mathrm{~min}$ antes do procedimento.

Após tricotomia ampla do membro pélvico direito e antissepsia do mesmo, foi realizada a abordagem cirúrgica à articulação coxofemoral direita, mediante incisão cutânea crânio-lateral. Após, incisou-se a fáscia lata e prosseguiu-se com a divulsão e afastamento dos músculos glúteos, tensor da fáscia lata e bíceps femoral para expor a articulação. A cabeça do fêmur foi inspecionada, não havendo sinais de erosões ou fraturas na mesma. O acetábulo foi acessado para remoção de resquícios do ligamento redondo, coágulos e debris teciduais. Após, realizou-se a redução da luxação. Pôde-se observar grande laceração da cápsula articular. A exposição do corpo do ílio foi obtida com o afastamento do músculo glúteo médio, utilizando um Afastador de Hohmann. Com uma perfuratriz e broca 3,0 mm, realizou-se um orifício no corpo do ílio, próximo à inserção do músculo reto femoral. $\mathrm{O}$ direcionamento da broca foi feito no sentido dorsolateral e ventro-medial (Figura 2a). Quatro fios de sutura absorvíveis de Polidioxanona número 2 (Figura 3 ) foram passados pelo túnel criado no corpo do ílio e tracionados com uma pinça 
hemostática curva (Figura 2b). Com uma broca de mesmo diâmetro, foi realizado outro orifício na base do trocânter maior do fêmur, transversal ao eixo ósseo longitudinal. Os fios que se encontravam dorsalmente ao corpo do ílio foram introduzidos no túnel femoral com o auxílio de um Passador de Fios. Os fios localizados ventralmente foram passados com uma pinça hemostática curva sobre a fossa inter-trocantérica e sob os músculos glúteos, configurando um padrão de sutura em oito (Figura 2c).

Promovendo uma ligeira rotação interna da cabeça femoral, os fios foram amarrados separadamente na porção caudal do trocânter maior, mantendo estável a articulação coxofemoral (Figura 2d). Prosseguiu-se com a rafia da fáscia lata e do músculo Tensor da Fáscia Lata ao Bíceps Femoral com fio multifilamentar absorvível de Ácido Poliglicólico 2-0 em padrão de sutura contínua, utilizando o mesmo material e tipo de sutura para a aproximação do tecido subcutâneo. A rafia cutânea foi realizada com Nylon Monofilamentar 3-0 em pontos isolados simples.

O membro não foi imobilizado. No pós-operatório imediato, foram aplicados Cetoprofeno 1 $\mathrm{mg} / \mathrm{kg} / \mathrm{IM}\left(\text { Ketofen }^{\circledR}\right)^{8}$ e Tramadol 2 mg/kg/IM (Cloridrato de Tramadol $\left.^{\circledR}\right)^{9}$. O paciente recebeu alta no mesmo dia com prescrição de Cetoprofeno $1 \mathrm{mg} / \mathrm{kg} /$ VO (Ketofen $\left.{ }^{\circledR}\right)^{8}$ a cada $24 \mathrm{~h}$ por quatro dias, Cloridrato de Tramadol $2 \mathrm{mg} / \mathrm{kg} / \mathrm{VO}\left(\operatorname{Tramal}^{\circledR}\right)^{9}$ a cada $8 \mathrm{~h}$ por 3 dias, limpeza diária da ferida cirúrgica com solução fisiológica, curativo com gaze e fita microporosa e repouso por 30 dias.

Só foi possível contatar o tutor por telefone e a revisão do paciente foi realizada 90 dias após a cirurgia, momento em que foi avaliada a deambulação e realizada a radiografia-controle com contenção química (Figura 1b).

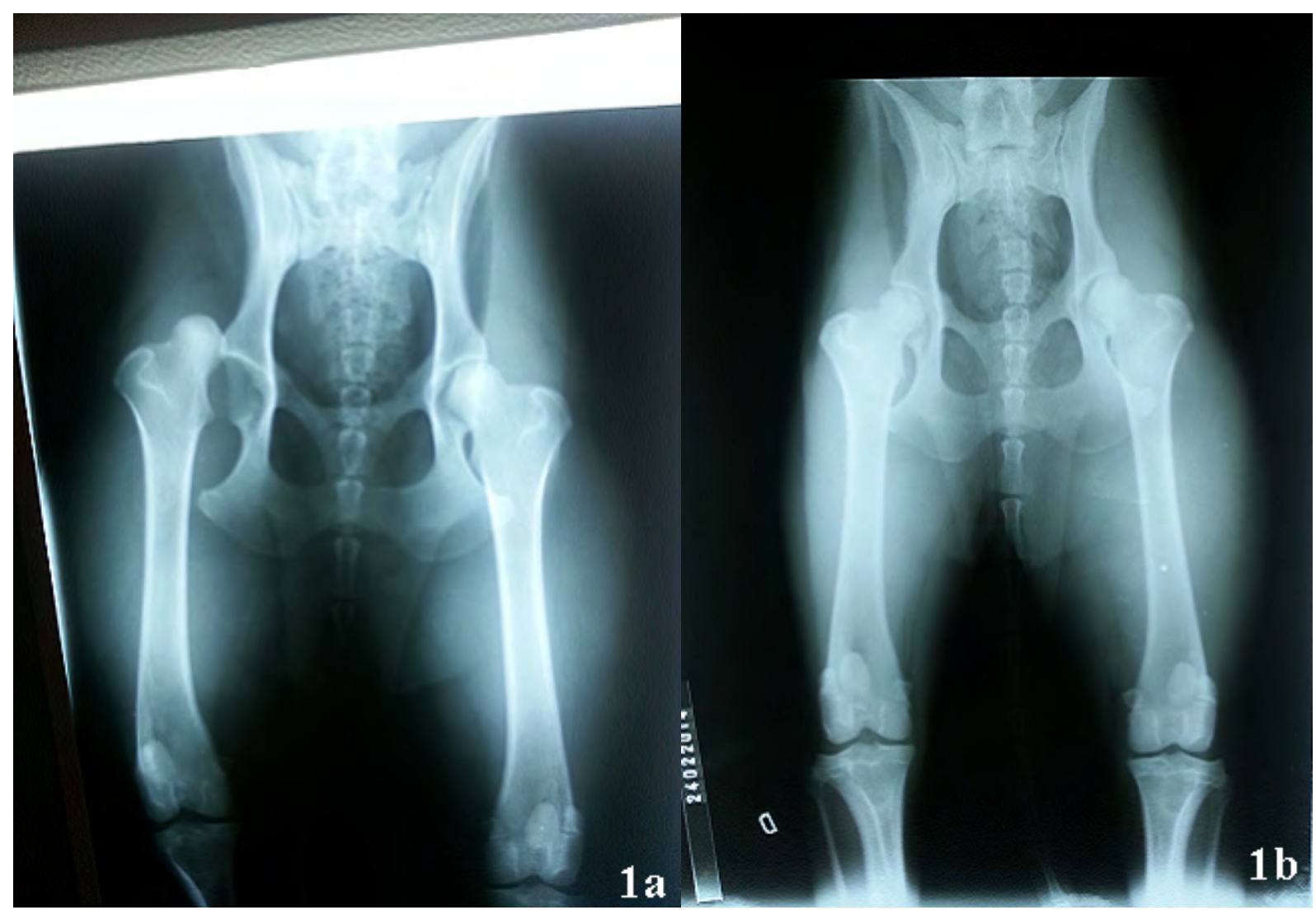

Figura 1. Radiografia pré-operatória na projeção V-D do paciente canino do presente relato, evidenciando a perda total do contato entre a superfície articular do acetábulo e cabeça femoral direitas, confirmando o diagnóstico de luxação coxofemoral (a). Radiografia-controle na projeção V-D do mesmo cão, realizada aos 90 dias de pós-operatório, onde podemos observar o reposicionamento anatômico da cabeça femoral direita em relação ao acetábulo, obtido após a redução cirúrgica da luxação. Há uma leve reação periosteal no corpo do ílio e atrofia da musculatura do membro pélvico direitos, em relação ao membro contralateral, apesar de o paciente estar apoiando normalmente o membro. 
L.H.Gaiga, J.A.T. Pigatto, C.L.Constantin \& T. Susin. 2016. Técnica de sutura ílio-femoral com fio de polidioxanona para estabilização da articulação coxofemoralem cão com luxação coxofemoral traumática. ActaScientiae Veterinariae.44(Suppl 1): 155.



Figura 2. Túnel ósseo realizado no corpo do ílio, com o auxílio de uma perfuratriz elétrica e guia de perfuração 3,0 mm (a). Fios de Polidioxanona passados pelo túnel confeccionado no corpo do ílio e tracionados com o auxílio de uma pinça hemostática curva (b). Fios passados por túnel ósseo confeccionado no trocânter maior e sob os músculos glúteos, configurando um padrão em " 8 " (c). Com o membro aduzido e ligeira rotação interna da cabeça femoral, fecham-se os nós separadamente na porção caudal do trocânter maior (d). As imagens apresentadas acima são de caráter ilustrativo e não se referem ao paciente do presente relato.

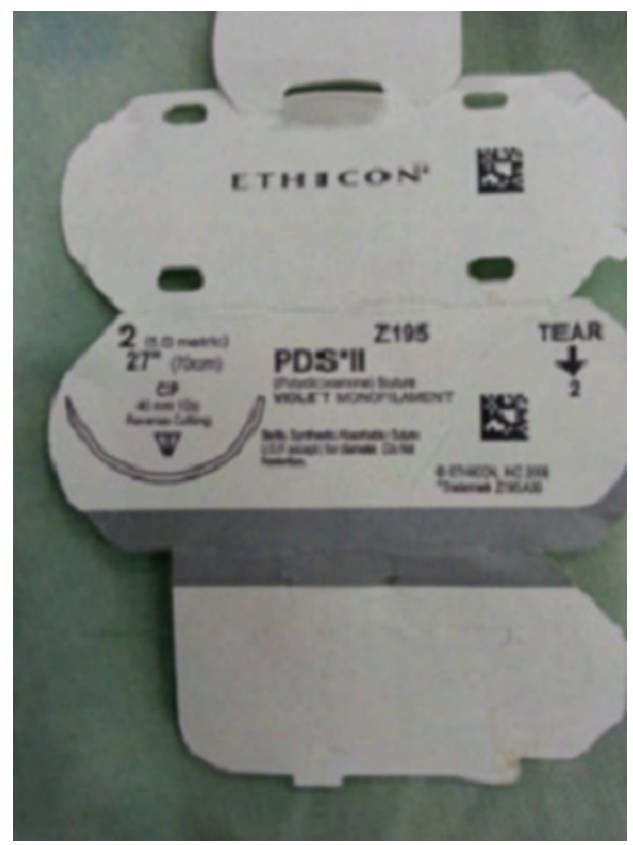

Figura 3. Fio monofilamentar absorvível de Polidioxanona número 2. 


\section{DISCUSSÃO}

O paciente do caso relatado foi vítima de acidente automobilístico, a causa mais comum de luxação coxofemoral [1,11]. Não houve, entretanto, lesões associadas, o que é frequente nessa afecção, principalmente o traumatismo torácico [13].

Os sinais apresentados de ausência de apoio e rotação interna são inespecíficos e podem estar associados a outras doenças, como por exemplo, fraturas de cabeça e colo femoral e de acetábulo [6,12]. O cão apresentava os sinais clínicos característicos de elevação e facilidade na palpação do trocânter maior e o aumento do espaço entre o mesmo e a tuberosidade isquiática $[5,9,13]$. Para confirmar o diagnóstico e descartar possíveis alterações concomitantes, radiografias simples da pelve e da articulação coxofemoral, nas projeções V-D e L-L, foram realizadas, como recomendado por alguns autores $[5,9,10,13]$.

Optou-se pela redução fechada logo que o paciente pôde ser anestesiado, para evitar maior dano aos tecidos moles e a degeneração da cartilagem articular [5,13]. A tipoia de Ehmer foi utilizada como método de imobilização temporária pelos mesmos motivos, mas devido ao elevado índice de falha do mesmo [2,3,13]. A abordagem crânio-lateral da articulação do quadril foi suficiente para a obtenção de uma boa exposição das estruturas envolvida e remoção de resquícios ligamentares e debris teciduais do acetábulo. $\mathrm{O}$ fato de a luxação ser recente e facilmente redutível eliminou a necessidade de expansão da abordagem $[6,13,14]$.

A técnica cirúrgica utilizada foi simples e facilmente exequível, não necessitando de material cirúrgico específico $[10,11]$. O procedimento teve a duração aproximada de $30 \mathrm{~min}$.

No presente relato, a cápsula articular estava lacerada, o que tornou inviável a rafia da mesma. A utilização da sutura ílio-femoral foi suficiente para promover a estabilidade da articulação [10,11]. Apesar da técnica não atuar em nenhuma estrutura de estabilização principal da articulação coxofemoral, não houve recidiva da luxação.

O fio de Polidioxanona foi escolhido com base nas suas características: o fato de ser absorvível, ter excelente proporção do comprimento em relação à força tênsil, mantendo-a por um longo período $[4,15]$. A Polidioxanona tem força tênsil maior e a mantém por mais tempo que a Poliglactina 910 $[4,15]$.

Durante o procedimento cirúrgico, pôde-se observar a facilidade de manipulação do fio [5]. Outra vantagem do fio de Polidioxanona é a sua mínima resposta tecidual, não gerando reação inflamatória do tipo corpo estranho [7].

As recomendações pós-operatórias foram estipuladas e seguidas de acordo com a literatura [10], não requerendo auxílio com a imobilização do membro, entretanto, o animal deveria apoiá-lo o quanto antes e o exercício deveria ser encorajado, porém, restrito. A não necessidade de imobilização pós-operatória é outro ponto favorável dessa técnica.

Conforme relatado pelo tutor, o cão começou a apoiar o membro três dias após a cirurgia. Aos 20 dias de pós-operatório caminhava sem claudicar, mas recolhia-o ao trote. Após 40 dias do procedimento, o tutor relatou apoio normal do membro pélvico direito ao caminhar e ao trote. Os resultados foram similares aos descritos por alguns autores [10,11].

Na revisão realizada aos 90 dias de pós-operatório, apesar da atrofia da musculatura do membro operado, o paciente apresentava apoio normal ao caminhar e trotar e pôde-se observar a total satisfação do tutor com o resultado da cirurgia.

Ao exame radiográfico, além da atrofia muscular, observou-se uma reação periosteal na região do corpo do ílio.

A utilização da técnica de sutura ílio-femoral com fio absorvível monofilamentar de Polidioxanona foi eficiente no tratamento da luxação coxofemoral craniodorsal no paciente do presente relato.

\section{MANUFACTURERS}

${ }^{1}$ Vetnil Ind. e Com. de Produtos Veterinários Ltda. Louveira, SP, Brazil.

${ }^{2}$ Laboratório Cristália. Itapira, SP, Brazil.

${ }^{3}$ Glicolabor Ind. Farmacêutica Ltda. Ribeirão Preto, SP, Brazil.

${ }^{4}$ Instituto BioChimico Ind. Farmacêutica Ltda. Itatiaia, RJ, Brazil.

${ }^{5}$ Laboratórios Bravet Ltda. Engenho Novo, RJ, Brazil.

${ }^{6}$ União Química Farmacêutica Nacional S.A. São Paulo, SP, Brazil.

${ }^{7}$ Ariston Ind. Química e Farmacêutica Ltda. São Paulo, SP, Brazil.

${ }^{8}$ Merial Saúde Animal Ltda. Campinas, SP, Brazil.

${ }^{9}$ Laboratórios Pfizer. Guarulhos, SP, Brazil.

Declaration of interest. The authors report no conflicts of interest. The authors alone are responsible for the content and writing of the paper. 


\section{REFERENCES}

1 Barbosa A.L.T. \& Schossler J.E.W. 2009. Luxação coxofemoral traumática em cães e gatos: estudo retrospectivo (1997-2006). Ciência Rural. 39(Suppl 6): 1823-1829.

2 Basher A.W.P. 1986. Coxofemoral luxation in the dog and cat. Veterinary Surgery. 15(Suppl 5): 356-362.

3 Bone D.L. 1984. Traumatic coxofemoral luxation in dogs: results of repair. Veterinary Surgery. 13: 263-270.

4 Boothe H.W. 2007. Fios de Sutura, Adesivos de Tecido, Grampeadores e Clipes Hemostáticos. In: Slatter D. (Ed). Manual de Cirurgia de Pequenos Animais. 3.ed. São Paulo: Manole, pp.235-243.

5 Fossum T.W. 2005. Management of Joint Diseases. In: Small Animal Surgery. St. Louis: Mosby, pp.943-957.

6 Harasen G. 2005. Coxofemoral luxations - part 2: surgical options. Canadian Veterinary Journal. 46(6): 546-547.

7 Hering F.L.O. \& Gabor S. 1993. Propriedades dos Fios de Sutura. In: Hering F.L.O., Gabor S. \& Rosenberg D. (Eds). Bases Técnicas e Teóricas de Fios e Suturas. São Paulo: Roca, pp.9-17.

8 Holsworth I.G. \& DeCamp C.E. 2007. Luxação Coxofemoral. In: Slatter D. (Ed). Manual de Cirurgia de Pequenos Animais. 3.ed. São Paulo: Manole, pp.2003-2008.

9 Kilic E., Ozaydin G., Atalen \& Baran V. 2002. Transposition of the sacrotuberous ligament for the treatment of coxofemoral luxations in dogs. Journal of Small Animal Practice. (43): 341-344.

10 Martini F.M., Simonazzi B. \& Bue M.D. 2001. Extra-articular absorbable suture stabilization of coxofemoral luxation in dogs. Veterinary Surgery. (30): 468-475.

11 Meiji B.P., Hazewinkel H.A.W. \& Nap R.C. 1992. Results of extra-articular stabilization following open reduction of coxofemoral luxation in dogs and cats. Journal of Small Animal Practice. (33): 320-326.

12 Moores A. 2006. Decision making in the management of hip luxations in dogs and cats. Companion Animal Practice. 28: 570-576.

13 Piermattei D.L. \& Flo G.L. 1999. A Articulação Coxofemoral. In: Manual de Ortopedia e Tratamento das Fraturas dos Pequenos Animais. São Paulo: Manole, pp.394-436.

14 Piermattei D.L. \& Greeley R.G. 1998. Abordagem à Articulação Coxofemoral através de uma Incisão Crânio-Lateral. In: Atlas de Abordagens Cirúrgicas aos Ossos do Cão e Gato. São Paulo: Manole, pp.132-133.

15 Smeak D.D. 1996. Escolha e Uso dos Materiais de Sutura atualmente disponíveis. In: Bojrab M.J. (Ed). Técnicas Atuais em Cirurgia de Pequenos Animais. 3.ed. São Paulo: Roca, pp.34-38. 